\title{
Mixed pairing symmetries and flux-induced spin current in mesoscopic superconducting loops with spin correlations
}

\author{
Guo-Qiao Zha, ${ }^{1,2,3}$ Lucian Covaci, ${ }^{3}$ F. M. Peeters, ${ }^{3}$ and Shi-Ping Zhou ${ }^{1,2}$ \\ ${ }^{1}$ Department of Physics, Shanghai University, Shanghai 200444, China \\ ${ }^{2}$ Shanghai Key Laboratory of High Temperature Superconductors, Shanghai University, Shanghai 200444, China \\ ${ }^{3}$ Departement Fysica, Universiteit Antwerpen, Groenenborgerlaan 171, B-2020 Antwerpen, Belgium
}

(Received 14 March 2015; revised manuscript received 18 May 2015; published 5 June 2015)

\begin{abstract}
We numerically investigate the mixed pairing symmetries in mesoscopic superconducting loops in the presence of spin correlations by solving the Bogoliubov-de Gennes equations self-consistently. The spatial variations of the superconducting order parameters and the spontaneous magnetization are determined by the band structure. When the threaded magnetic flux turns on, the charge and spin currents both emerge and depict periodic evolution. In the case of a mesoscopic loop with dominant triplet $p_{x} \pm i p_{y}$-wave symmetry, a slight change of the chemical potential may lead to novel flux-dependent evolution patterns of the ground-state energy and the magnetization. The spin-polarized currents show pronounced quantum oscillations with fractional periods due to the appearance of energy jumps in flux, accompanied with a steplike feature of the enhanced spin current. Particularly, at some appropriate flux, the peaks of the zero-energy local density of states clearly indicate the occurrence of the odd-frequency pairing. In the case of a superconducting loop with dominant singlet $d_{x^{2}-y^{2}}$-wave symmetry, the spatial profiles of the zero-energy local density of states and the magnetization show spin-dependent features on different sample diagonals. Moreover, the evolution of the flux-induced spin current always exhibits an $h c / e$ periodicity.
\end{abstract}

DOI: 10.1103/PhysRevB.91.214504

PACS number(s): 74.20.Rp, 74.25.Ha, 74.78.Na

\section{INTRODUCTION}

Recently, the flux-dependent periodic evolution of supercurrent in mesoscopic $s$ - or $d$-wave superconducting loops has drawn a lot of attention [1-11], which is a valuable probe into the mechanism of superconductivity and is the basis for many applications. For superconductors with spin-singlet pairing, the doubling or breaking of well-known $h c / 2 e$-flux periodicity has been predicted due to the lifted degeneracy in energy and the offset of the transition between different current-carrying states. Another system which may support novel flux periodicity is the spin-triplet $p$-wave superconductor [12]. For $\mathrm{Sr}_{2} \mathrm{RuO}_{4}$, which is a prime candidate for the topological superconductivity, it has been proposed that the antiferromagnetic spin fluctuation may lead to the spin-triplet superconducting pairing symmetry [13,14]. Recent cantilever magnetometry measurements of annular mesoscopic samples of $\mathrm{Sr}_{2} \mathrm{RuO}_{4}$ have revealed evidence for the existence of half-quantum vortices in this material [15]. Furthermore, unconventional quantum oscillations with distinct flux periods have been reported in mesoscopic superconducting rings of $\mathrm{Sr}_{2} \mathrm{RuO}_{4}[16]$.

Notice that, if the inversion symmetry is broken by the space inhomogeneity of the order parameter, mixing of the singlet and triplet states becomes possible in the superconducting system with lifted spin degeneracy $[17,18]$. It has been predicted that an additional $p$-wave component can be generated by the surface induced spin-orbit coupling in the case of $d$-wave superconductors [19,20]. Additionally, for a square lattice system with a nearest-neighbor attractive interaction, superconducting states with various symmetries can be found by changing the band structure (i.e., the shape of the Fermi surface) [21,22]. Due to the proximity effect, the mixed parity pairing and spontaneous spin current have been observed near the interface between unconventional superconductors and ferromagnets $[23,24]$. In particular, a potential barrier modulating the electronic density near the edge of the system can lead to a nonunitary superconducting state close to the boundary where spin-singlet pairing coexists with the dominant triplet order, and the spin polarization and accumulation may appear in a system without the proximity coupling to an exotic one [25]. These imply that interesting phenomena related to the mixed pairing symmetries and the periodic evolution of charge and spin currents may be produced in mesoscopic superconducting loops.

In the present work, we provide a careful insight into the mixed pairing states and corresponding flux periodic evolution of current in mesoscopic superconducting loops. We perform a microscopic self-consistent calculation by solving the Bogoliubov-de Gennes (BdG) equations [26] in real space. It has been known that the on-site repulsion interaction within the mean-field approximation is essential to generate the spin order in high-temperature superconductors due to competing antiferromagnetism and $d$-wave superconductivity [27-30]. We will introduce the repulsive on-site interaction among the electrons into the model Hamiltonian to generate the spin correlation, which has not been considered in mesoscopic loop systems yet. The effect of the band structure will be applied to tune the stable spin-triplet ( $p$-wave) and spin-singlet ( $d$ - or $s$-wave) superconducting states as well as states with their coexistence within the same type of interaction. For an appropriate chemical potential, the mixed pairing symmetry of order parameters can be realized in the same material. Our numerical analysis focuses on two types of the square loop with dominant ( $\left.p_{x} \pm i p_{y}\right)$-wave or $d_{x^{2}-y^{2}}$-wave symmetry. The local magnetization and zero-energy local density of states (LDOS) are demonstrated correspondingly. When a magnetic flux is turned on, the charge and spin currents show up with novel evolution patterns. 
The paper is organized as follows. In Sec. II we present our theoretical formalism. In Secs. III and IV we discuss the results obtained for the mixed pairing states and flux-dependent current evolution in two cases of the superconductor with dominant $p$ - or $d$-wave symmetry. Our results are summarized in Sec. V.

\section{THEORETICAL APPROACH}

To investigate the flux periodicity in a mesoscopic superconducting loop whose edges are oriented parallel to the [100] and [010] directions, we start with the pairing Hamiltonian by assuming nearest-neighbor attraction $V$ for superconducting pairing:

$$
\begin{aligned}
\hat{H}= & -\sum_{\langle\mathbf{i j}\rangle, \sigma} t_{\mathbf{i j}} \exp \left(i \varphi_{\mathbf{i j}}\right) c_{\mathbf{i} \sigma}^{\dagger} c_{\mathbf{j} \sigma}+\sum_{\mathbf{i}, \sigma}\left(U\left\langle n_{\mathbf{i} \sigma}\right\rangle-\mu\right) c_{\mathbf{i} \sigma}^{\dagger} c_{\mathbf{i} \sigma} \\
& +V \sum_{\langle\mathbf{i j}\rangle}\left(\Delta_{\mathbf{i j}} c_{\mathbf{i} \uparrow}^{\dagger} c_{\mathbf{j} \downarrow}^{\dagger}+\Delta_{\mathbf{i j}}^{*} c_{\mathbf{j} \downarrow} c_{\mathbf{i} \uparrow}\right)
\end{aligned}
$$

where $t_{\mathbf{i j}}=t$ are the nearest-neighbor hopping integrals. $c_{\mathbf{i} \sigma}$ $\left(c_{\mathbf{i} \sigma}^{\dagger}\right)$ are destruction (creation) operators for the electron of spin $\sigma(\sigma=\uparrow$ or $\downarrow)$. $U$ represents the on-site repulsion interaction. $n_{\mathbf{i} \sigma}=c_{\mathbf{i} \sigma}^{\dagger} c_{\mathbf{i} \sigma}$ is the number operator, and $\mu$ is the chemical potential determining the averaged electron density $\bar{n}$. The Peierls phase factor is given by $\varphi_{\mathbf{i j}}=2 \pi / \Phi_{0} \int_{r_{\mathrm{i}}}^{r_{\mathbf{j}}} \mathbf{A}(\mathbf{r}) \cdot d \mathbf{r}$ with the flux quantum $\Phi_{0}=h c / e$. We choose a vector potential of the form $\mathbf{A}(\mathbf{r})=(y,-x, 0) \Phi /\left[2 \pi\left(x^{2}+y^{2}\right)\right]$, yielding a flux threading the hole with no magnetic field penetrating the superconductor, where $\phi=(e / h c) \Phi$ measures the flux in units of $h c / e$. Here we have introduced the pairing amplitude on a bond $\Delta_{\mathbf{i j}}=\left\langle c_{\mathbf{i} \uparrow} c_{\mathbf{j} \downarrow}\right\rangle$. Using the Bogoliubov transformation, $c_{\mathbf{i} \sigma}=\sum_{n}\left[u_{\mathbf{i} \sigma}^{n} \gamma_{n \sigma}-\sigma v_{\mathbf{i} \sigma}^{n *} \gamma_{n \bar{\sigma}}^{\dagger}\right]$, the Hamiltonian in Eq. (1) can be diagonalized by solving the resulting $\mathrm{BdG}$ equations self-consistently:

$$
\sum_{\mathbf{j}}^{N}\left(\begin{array}{cc}
\mathcal{H}_{\mathbf{i j} \sigma} & \Delta_{\mathbf{i j}} \\
\Delta_{\mathbf{i j}}^{\dagger} & -\mathcal{H}_{\mathbf{i j} \bar{\sigma}}^{*}
\end{array}\right)\left(\begin{array}{c}
u_{\mathbf{j} \sigma}^{n} \\
v_{\mathbf{j} \bar{\sigma}}^{n}
\end{array}\right)=E_{n}\left(\begin{array}{c}
u_{\mathbf{i} \sigma}^{n} \\
v_{\mathbf{i} \bar{\sigma}}^{n}
\end{array}\right),
$$

where $\mathcal{H}_{\mathbf{i j} \sigma}=-t_{\mathbf{i j}} \exp \left(i \varphi_{\mathbf{i j}}\right)+\left[U\left\langle n_{\mathbf{i} \sigma}\right\rangle-\mu\right] \delta_{\mathbf{i j}}$. With the open boundary conditions (for which the wave function vanishes on the inner and outer boundaries of the loop) we can get the eigenvalues $\left\{E_{n}\right\}$ with eigenvectors $\left\{u_{\mathbf{i}}^{n}, v_{\mathbf{i}}^{n}\right\}$. The self-consistent conditions are

$$
\begin{aligned}
\left\langle n_{\mathbf{i} \uparrow}\right\rangle & =\sum_{n=1}^{2 N}\left|u_{\mathbf{i}}^{n}\right|^{2} f\left(E_{n}\right), \\
\left\langle n_{\mathbf{i} \downarrow}\right\rangle & =\sum_{n=1}^{2 N}\left|v_{\mathbf{i}}^{n}\right|^{2}\left[1-f\left(E_{n}\right)\right], \\
\Delta_{\mathbf{i j}} & =\sum_{n=1}^{2 N} u_{\mathbf{i}}^{n} v_{\mathbf{j}}^{n *}\left[1-f\left(E_{n}\right)\right],
\end{aligned}
$$

where $f\left(E_{n}\right)=\left(e^{E_{n} / k_{B} T}+1\right)^{-1}$ is the Fermi-Dirac distribution function. From the order parameter $\Delta_{\mathrm{ij}}$, it is possible to build the spin-singlet $(S)$ and spin-triplet $(T)$ pairing amplitudes, given by [22-25]

$$
\begin{aligned}
\Delta_{\mathrm{ij}}^{S} & =\left(\Delta_{\mathrm{ij}}+\Delta_{\mathrm{ji}}\right) / 2, \\
\Delta_{\mathrm{ij}}^{T} & =\left(\Delta_{\mathrm{ij}}-\Delta_{\mathrm{ji}}\right) / 2 .
\end{aligned}
$$

Then the extended $s-, d_{x^{2}-y^{2-}}, p_{x^{-}}$, and $p_{y}$-wave symmetry can be defined, respectively, at site $\mathbf{i}$ as

$$
\begin{aligned}
\Delta_{s}(\mathbf{i}) & =\left(\Delta_{\mathbf{i}+\mathbf{e}_{\mathbf{x}}, \mathbf{i}}^{S}+\Delta_{\mathbf{i}-\mathbf{e}_{\mathbf{x}}, \mathbf{i}}^{S}+\Delta_{\mathbf{i}, \mathbf{i}+\mathbf{e}_{\mathbf{y}}}^{S}+\Delta_{\mathbf{i}, \mathbf{i}-\mathbf{e}_{\mathbf{y}}}^{S}\right) / 4, \\
\Delta_{d}(\mathbf{i}) & =\left(\Delta_{\mathbf{i}+\mathbf{e}_{\mathbf{x}}, \mathbf{i}}^{S}+\Delta_{\mathbf{i}-\mathbf{e}_{\mathbf{x}}, \mathbf{i}}^{S}-\Delta_{\mathbf{i}, \mathbf{i}+\mathbf{e}_{\mathbf{y}}}^{S}-\Delta_{\mathbf{i}, \mathbf{i}-\mathbf{e}_{\mathbf{y}}}^{S}\right) / 4, \\
\Delta_{p_{x}}(\mathbf{i}) & =\left(\Delta_{\mathbf{i}+\mathbf{e}_{\mathbf{x}}, \mathbf{i}}^{T}-\Delta_{\mathbf{i}-\mathbf{e}_{\mathbf{x}}, \mathbf{i}}^{T}\right) / 2, \\
\Delta_{p_{y}}(\mathbf{i}) & =\left(\Delta_{\mathbf{i}, \mathbf{i}+\mathbf{e}_{\mathbf{y}}}^{T}-\Delta_{\mathbf{i}, \mathbf{i}-\mathbf{e}_{\mathbf{y}}}^{T}\right) / 2,
\end{aligned}
$$

where $\mathbf{e}_{\mathbf{x}(\mathbf{y})}$ denotes the unit vector along the $x(y)$ direction. As regards the spin-triplet pairing, we only choose the case with $S_{z}=0$ for simplicity. The bond current densities $J_{\mathbf{i j} \sigma}$ from lattice site $\mathbf{i}$ to $\mathbf{j}$ carried by spin-up and spin-down electrons are given by

$$
\begin{array}{r}
J_{\mathbf{i} \mathbf{} \uparrow}=-4 \frac{e t}{\hbar c} \sum_{n} \operatorname{Im}\left[u_{\mathbf{j}}^{n} u_{\mathbf{i}}^{n *} f\left(E_{n}\right) \exp \left(i \varphi_{\mathbf{i j}}\right)\right], \\
J_{\mathbf{i} \downarrow}=-4 \frac{e t}{\hbar c} \sum_{n} \operatorname{Im}\left[v_{\mathbf{j}}^{n *} v_{\mathbf{i}}^{n}\left(1-f\left(E_{n}\right)\right) \exp \left(i \varphi_{\mathbf{i j}}\right)\right] .
\end{array}
$$

The current $J_{\mathbf{i}}$ is defined as the average of the neighboring bond currents. We can determine the local charge and spin current $[23,25] J_{c}(\mathbf{i})=J_{\mathbf{i} \uparrow}+J_{\mathbf{i} \downarrow}$ and $J_{s}(\mathbf{i})=J_{\mathbf{i} \uparrow}-J_{\mathbf{i} \downarrow}$, respectively. The total energy $F$ of the system is given by [31]

$$
F=\langle\hat{H}\rangle=\mathrm{KE}+\sum_{\mathbf{i}}\left[2 U n_{\mathbf{i} \downarrow} n_{\mathbf{i} \uparrow}-\mu n_{\mathbf{i}}-\sum_{\mathbf{j}} \frac{\left|\Delta_{\mathbf{i j}}\right|^{2}}{V}\right],
$$

where $\mathrm{KE}$ is the kinetic energy of the system:

$$
\mathrm{KE}=-\frac{1}{2} \sum_{\mathbf{i j}, n} t_{\mathbf{i j}} \exp \left(i \varphi_{\mathbf{i j}}\right)\left(v_{\mathbf{i}}^{n} v_{\mathbf{j}}^{n *}-u_{\mathbf{j}}^{n} u_{\mathbf{i}}^{n *}\right) \tanh \left(\frac{E_{n}}{2 k_{B} T}\right) .
$$

The LDOS can be written as

$$
\rho_{\mathbf{i} \sigma}(E)=\sum_{n, \sigma}\left[\left|u_{\mathbf{i} \sigma}^{n}\right|^{2} \delta\left(E_{n}-E\right)+\left|v_{\mathbf{i} \sigma}^{n}\right|^{2} \delta\left(E_{n}+E\right)\right],
$$

where the Dirac delta function $\delta(x)$ is taken as $\Gamma / \pi\left(x^{2}+\Gamma^{2}\right)$ with the quasiparticle damping $\Gamma=0.01$. The LDOS is proportional to the local differential tunneling conductance which could be measured in a low-temperature scanning tunneling microscopic (STM) experiment.

Throughout this work, the distance is measured in units of the lattice constant $a$, the energy in units of $t$, the magnetic flux in units of $\Phi_{0}=h c / e$, and the current density in units of $J_{0}=$ $e t / \hbar c$. In the numerical calculations, we take $k_{B}=a=t=1$ for simplicity. In what follows, we focus on the mesoscopic superconducting square loop with an outer size $N_{x} \times N_{y}=$ $40 \times 40$ and an arm width $w=14$, which is threaded by a magnetic field $\Phi$ in the hole. For an appropriate initial set of parameters $n_{\mathbf{i} \sigma}$ and $\Delta_{\mathbf{i j}}$, the Hamiltonian is numerically diagonalized and the electron wave functions obtained are used to calculate the new parameters for the next iteration step. The calculations are repeated until the difference in the order parameters between two consecutive iterations is less than $10^{-6}$. A ground state has the lowest total energy among those of stable states evaluated from different possible initial parameters. 
As known, the studies on the extended Hubbard model [21,22] have shown that a $d_{x^{2}-y^{2}}$-wave superconducting state is stabilized near half-filling $\mu \sim 0$, while the extended $s$-wave state is favored near the filling $|\mu| \sim 2.5 t$. In the region between $d$ - and $s$-wave states, the spin-triplet $p_{x} \pm i p_{y^{-}}$ wave superconducting state appears. In the present work, we use this result to choose the parameters to realize the dominant $p_{x} \pm i p_{y}$ - or $d_{x^{2}-y^{2}}$-wave pairing. Near the boundary between different pairing symmetries, the states where the spin-singlet and the spin-triplet order parameters coexist may be found.

\section{MESOSCOPIC LOOP WITH DOMINANT $p$-WAVE SYMMETRY}

In this section, we investigate the mesoscopic superconducting loop with a dominant spin-triplet $p_{x} \pm i p_{y}$-wave symmetry at zero temperature. Here we choose $\mu=-1.6$, which is in the window of stability for the $p$-wave order. The interaction strengths are set as $V=2.5$ and $U=1.5$. In the mesoscopic size case, we find that the spin-singlet $d$ - and $s$-wave components of the order parameter can appear at the same time, i.e., the mixed pairing symmetries are allowed here. The underlying physics can be qualitatively explained as follows. In the present finite system, translational symmetry is broken, and parity of the Cooper pair is no longer a good quantum number. Then, the mixed parity state can be realized. On the other hand, the spin rotational symmetry is broken due to the presence of the spin correlation, and spin-singlet and spin-triplet parings are mixed with each other. Note that the $d$-wave superconductivity does not emerge when $\mu \leqslant-1.8$. Further decreasing the electron density, the $p$-wave pairing gradually disappears and only an $s$-wave order can exist in our mesoscopic system. Figure 1 shows the spatial profiles of order parameters for $p_{x}$-wave (a), $p_{y}$-wave (b), $d$-wave (c), and extended $s$-wave (d) pairings in the square loop when the threaded magnetic flux $\Phi=0$. It is seen that the $p_{x}\left(p_{y}\right)$ order only appears in arms of the loop along the $x(y)$ direction. $\Delta_{p_{x}}$ $\left(\Delta_{p_{v}}\right)$ almost takes on the constant magnitude inside the sample and is suppressed near the inner and outer boundaries as well as on the sample diagonals. The subdominant $s$-wave order is mainly localized at the inner and outer corners. The induced $\Delta_{d}$ component is much smaller than the case of dominant $p$-wave state, and the $d$-wave superconducting order is enhanced near the sample edge. Inside the sample, the spin-singlet orders both show oscillatory behaviors due to the finite-size effect.

To further characterize the coexisting states in this mesoscopic system, we analysis the spontaneous magnetic moment and the corresponding LDOS at zero threaded flux. It has been predicted that the emergency of the mixed parity phase may be accompanied by the appearance of the finite spin polarization [25]. Also, the STM experiment with atomic-scale resolution is well suited for probing surfaces and inhomogeneous systems. In Fig. 2, we depict the two-dimensional contour plots of the zero-energy LDOS $\rho_{\uparrow}(E=0)$ (a), $\rho_{\downarrow}(E=0)$ (b), and $\rho_{\uparrow \downarrow}(E=0)$ (c) for the cases of spin-up, spin-down, and total spin, respectively. The profile of the magnetization $m_{\mathbf{i}}=\left(n_{\mathbf{i} \uparrow}-n_{\mathbf{i} \downarrow}\right) / 2$ is given in Fig. 2(d). We can see that the zero-energy modes for spin-up and spin-down electrons are
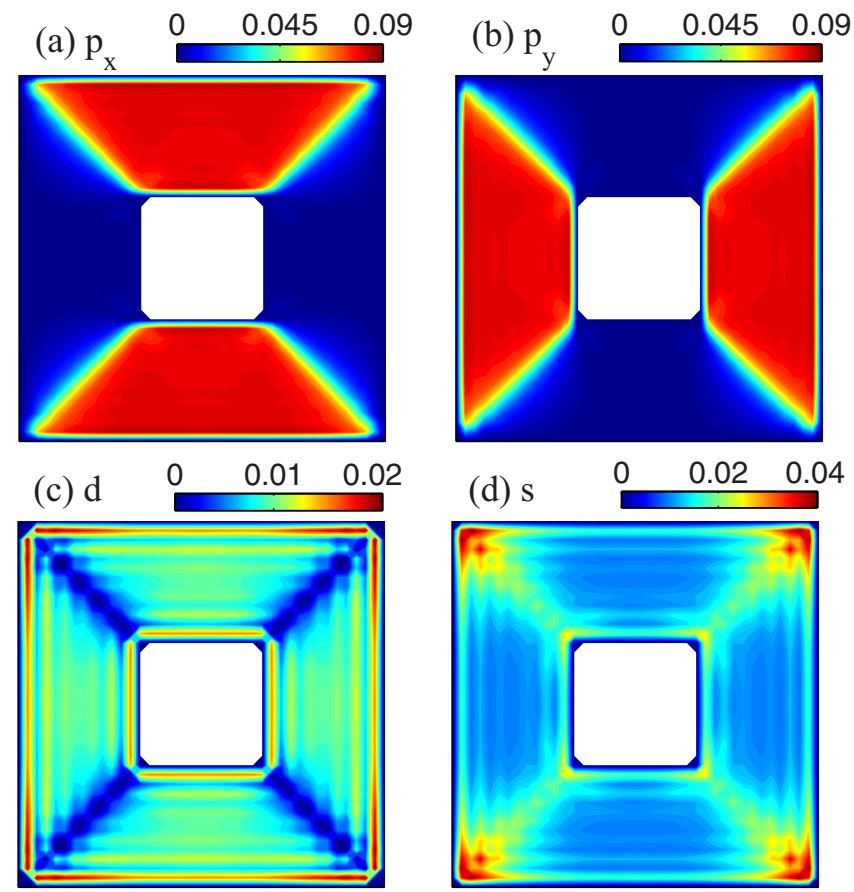

FIG. 1. (Color online) Contour plots of $p_{x}$-wave (a), $p_{y}$-wave (b), $d$-wave (c), and extended $s$-wave (d) pairings for a square $40 \times 40$ loop with an arm width $w=14$ at zero flux. The calculation is performed with $V=2.5, U=1.5, \mu=-1.6$, and the temperature $T=0$.

slightly different and the zero-energy peaks mainly emerge in the diagonal directions in Figs. 2(a) and 2(b). The zero-bias peak conductance, together with the weak oscillation inside the sample, may reflect the appearance of a subdominant extended $s$-wave component in our system, analogous to the order parameter profile in Fig. 1(d). Simultaneously, a spontaneous magnetic moment appears near the corners of the sample with a nonunitary superconducting state [see Fig. 2(d)], similar to the case of a slab geometry with a finite surface barrier in the absence of a proximity effect due to the modulated electronic density [25].

It is important to note that the presence of the zeroenergy peaks on the sample diagonals in the LDOS map may also imply interesting pairing symmetry with respect to the frequency in our mesoscopic system with strong spin correlations. In accordance with the Fermi-Dirac statistics, there exist two possibilities of odd-frequency pairing, i.e., spinsinglet odd-parity and spin-triplet even-parity pairings [32], due to the breakdown of the translational and spin-rotational invariances. It has been shown that odd-frequency spin-triplet even-parity (OTE) pairing highly influences the density of states of quasiparticles, accompanied with a zero-energy peak structure [33]. The appearance of odd-frequency pairing in the present system can be clearly demonstrated in the slightly decreased $|\mu|$ case, which will be discussed in a subsequent part of this section.

When the threaded magnetic flux turns on, there are persistent flows of both spin-up and spin-down electrons. Normally, this gives rise to well-known persistent charge current, and its flux periodic evolution has been widely 

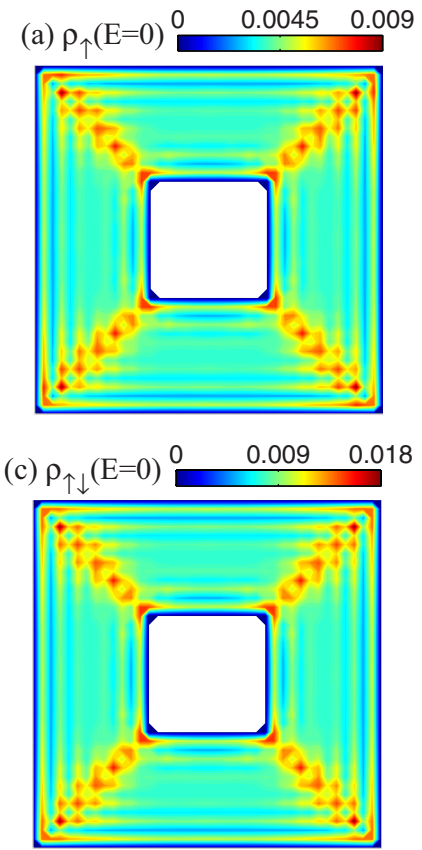

FIG. 2. (Color online) Contour plots of zero-energy local density of states $\rho_{\uparrow}(E=0)(\mathrm{a}), \rho_{\downarrow}(E=0)(\mathrm{b}), \rho_{\uparrow \downarrow}(E=0)(\mathrm{c})$, and magnetization $m_{\mathbf{i}}=\left(n_{\mathbf{i} \uparrow}-n_{\mathbf{i} \downarrow}\right) / 2$ (d) for a square $40 \times 40$ loop with $w=14$ at $\Phi / \Phi_{0}=0$ when $\mu=-1.6$

discussed. Once the persistent charge current is spin polarized, a nonzero persistent spin current can be generated. Next, we would like to discuss the flux-dependent evolution of the charge and spin currents in our studied system with mixed pairing. Figure 3 shows the total energy (a) and the persistent spin current $J_{s}$ (the solid curve) as well as the spin-polarized currents $J_{\uparrow}$ (the dashed curve) and $J_{\downarrow}$ (the dash-dotted curve)

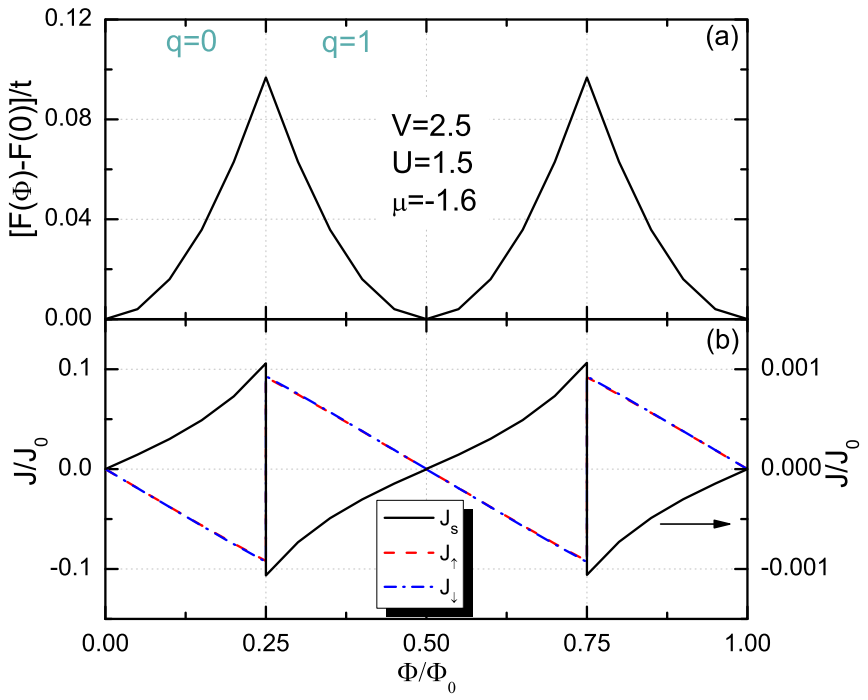

FIG. 3. (Color online) Total energy (a) and spin current $J_{s}$ (righthand scale) and spin-polarized currents $J_{\uparrow}$ and $J_{\downarrow}$ (the left-hand scale) (b) for a square $40 \times 40$ loop with $w=14$ as a function of magnetic flux $\Phi$ when $\mu=-1.6 . q$ is the winding number of the order parameter. (b) as a function of magnetic flux $\Phi$ in the mesoscopic square loop. In order to demonstrate clearly the flux evolution of the spin current, the magnitude of $J_{s}$ is always given by the right-hand scale. For the chosen parameter values, the total energy reaches local minima at $\Phi=n \Phi_{0} / 2$ (here, $n$ denotes an integer) and exhibits exactly a $\Phi_{0} / 2$ periodic evolution. Correspondingly, the phase transitions between the states with even and odd winding numbers $q$ of the order parameter with respect to $\Phi=(2 n+1) \Phi_{0} / 4$ render that the spin-polarized currents develop fully the $\Phi_{0} / 2$-flux periodicity. In general, the spin-polarized currents and then the spin current show saw-tooth-like patterns in flux. Between the flux values where the current jumps, the currents with standard linear behavior for spin-up and spin-down electrons are almost overlap in the present case, resulting in weak spin currents at finite flux. When the flux is enlarged from zero, $J_{s}$ first appears and then keeps increasing. At the transiting point $\Phi=\Phi_{0} / 4$, the spin current changes sign and flows oppositely. In order to understand better the spin current distribution, Fig. 4 depicts the spatial variations of the spin-polarized currents $J_{\uparrow}$ (a) and $J_{\downarrow}$ (b) as well as the charge current $J_{c}$ (c) and spin current $J_{s}$ (d) at $\Phi / \Phi_{0}=0.25$ in the $q=0$ sector. One can clearly see that the profiles of $J_{\uparrow}$ and $J_{\downarrow}$ are nearly the same at the arm center, while remaining different in the diagonal directions. It is noted that the origin of the spin polarization is in the appearance of the regime of local magnetization. As a consequence, the spin current shows pronounced oscillations near the loop diagonals [see Fig. 4(d)].

Notice that the spatial profiles of the magnetization and the zero-energy LDOS almost keep the same as the zero flux case in Fig. 2 with increasing $\Phi$ for $\mu=-1.6$. Meanwhile, the total

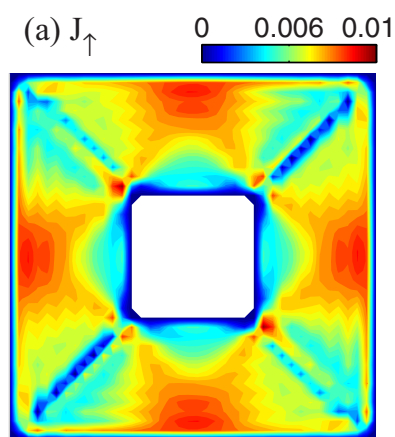

$\begin{array}{llll}\text { (c) } \mathrm{J}_{\mathrm{c}} & 0 & 0.01 & 0.02\end{array}$

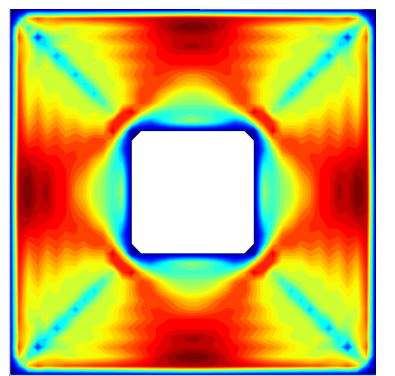

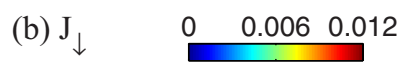

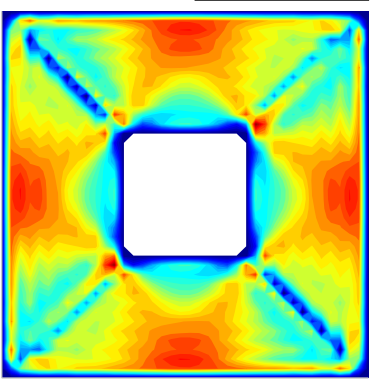

(d) $\mathrm{J}$

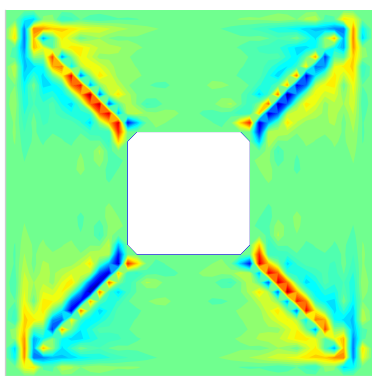

FIG. 4. (Color online) Contour plots of spin-polarized currents $J_{\uparrow}$ (a) and $J_{\downarrow}$ (b) as well as the charge current $J_{c}$ (c) and spin current $J_{s}$ (d) for a square $40 \times 40$ loop with $w=14$ at $\Phi / \Phi_{0}=0.25$ in the $q=0$ sector when $\mu=-1.6$. 


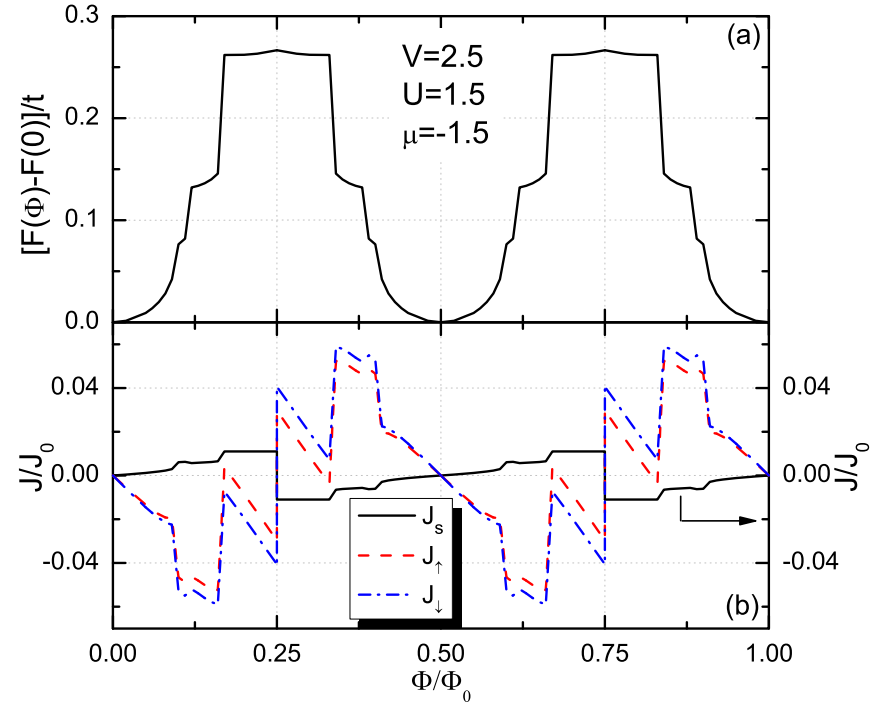

FIG. 5. (Color online) Total energy (a) and spin current $J_{s}$ (righthand scale) and spin-polarized currents $J_{\uparrow}$ and $J_{\downarrow}$ (the left-hand scale) (b) for a square $40 \times 40$ loop with $w=14$ as a function of $\Phi$. The calculation is performed with $V=2.5, U=1.5, \mu=-1.5$, and the temperature $T=0$.

energy shows a standard parabolic behavior with flux in every $q$ sector. However, this shape does not remain at finite flux for a reduced $|\mu|$ due to a stronger effect of the band structure on the symmetry of superconducting states. Thus, more novel phenomena related to flux periodic evolution may be present.

As a representative example, Fig. 5 displays the flux evolution of the total energy (a) and currents $J_{\uparrow}, J_{\downarrow}$, and $J_{s}$ (b) for a slightly changed $\mu=-1.5$. One can easily see that, as compared to the case in Fig. 3, the energy and the circulating current still oscillate periodically as a function of flux, while the evolution patterns behave more complicated. In Fig. 5(a), the ground-state energy forms parabolas with a global minimum at integer and half-integer values of $\Phi$, which is identical to the previous case with a large $|\mu|$. Interestingly, there appear additional energy parabolas in $F(\Phi)$ between these flux values, and the energy increases or decreases stepwise between different parabolas. As a consequence, the spin-polarized currents $J_{\uparrow(\downarrow)}(\Phi)$ in Fig. 5(b) are discontinuous at the flux values where the energy jumps [see the dashed and dash-dotted curves], which may support the appearance of unconventional quantum oscillations with fractional flux periodicity [16]. Notice that the flux regimes belonging to different energy parabolas are not distributed equally and then peculiar sawtooth patterns of flux-dependent charge current show up. Remarkably, there exists a pronounced difference between $J_{\uparrow}$ and $J_{\downarrow}$, giving rise to stronger spin currents in contrast to the case in Fig. 3(b). As shown by the black solid curve in Fig. 5(b), $J_{s}$ emerges when the flux is threaded and increases monotonously at small $\Phi$. Between the flux values where the energy jumps, $J_{\uparrow}$ and $J_{\downarrow}$ show standard linear behavior and changes synchronously. Therefore, the magnitude of $J_{s}$ almost takes a constant value, i.e., the step feature of the spin current appears at large flux. As a result, the
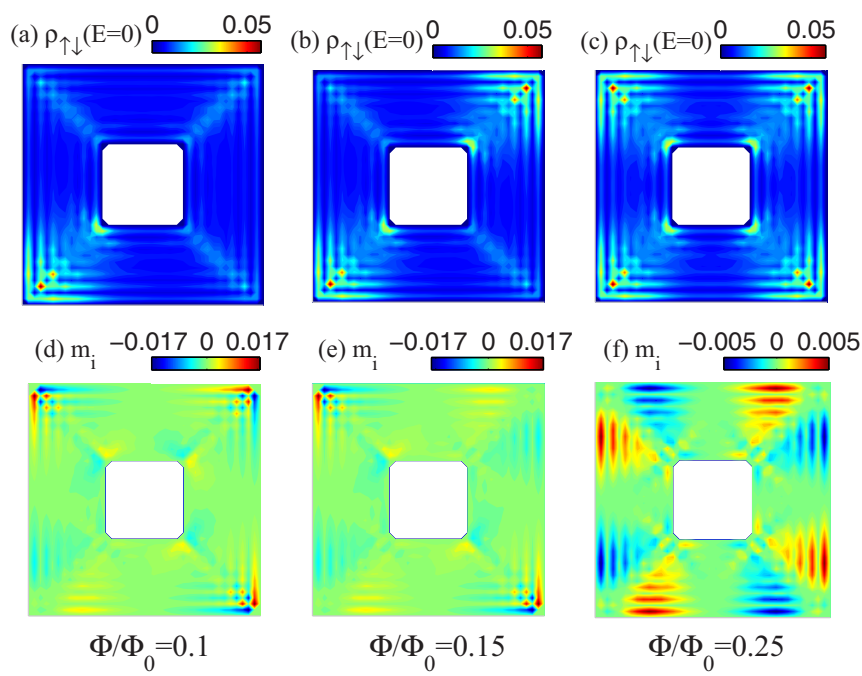

FIG. 6. (Color online) Contour plots of zero-energy local density of states $\rho_{\uparrow \downarrow}(E=0)(\mathrm{a}-\mathrm{c})$ and magnetization $m_{\mathbf{i}}(\mathrm{d}, \mathrm{e})$ for a square $40 \times 40$ loop with $w=14$ at $\Phi / \Phi_{0}=0.1$ (left panels), $\Phi / \Phi_{0}=0.15$ (middle panels), and $\Phi / \Phi_{0}=0.25$ (right panels) when $\mu=-1.5$.

persistent spin current exhibits two flux-dependent features in the periodic oscillation.

Notably, novel flux evolution of the magnetization may take place when the energy jumps between different parabolas in the ground state. In Fig. 6, we depict the zero-energy LDOS (the upper panels) $\rho_{\uparrow \downarrow}(E=0)$ and the magnetization $m_{\mathbf{i}}$ (the bottom panels) for the square loop at the flux values $\Phi / \Phi_{0}=$ 0.1 (left panels), $\Phi / \Phi_{0}=0.15$ (middle panels), and $\Phi / \Phi_{0}=$ 0.25 (right panels) belonging to different energy parabolas. We only show the LDOS for the total spin. At zero flux, the system has almost the same spatial distribution of $m_{\mathbf{i}}$ as the case in Fig. 2(d). This profile remains with increasing flux until $\Phi / \Phi_{0} \simeq 0.09$ where the energy first jumps. As seen in Fig. 6(d) for $\Phi / \Phi_{0}=0.1$, there is a distinct suppression of magnetization on the left-down diagonal of the sample. Further increasing $\Phi$, two other patterns of the magnetization shown in Figs. 6(e) and 6(f) can be found in different energy parabolas. At the transition point $\Phi / \Phi_{0}=0.25$ from the $q=0$ state to the $q=1$ state, we can clearly see that the magnetization is suppressed on all diagonals in Fig. 6(f).

Interestingly, the disappearance of the magnetization is accompanied by an enhancement of the zero-energy peaks of LDOS [see Figs. 6(a)-6(c)]. We may attribute this to the generation of odd-frequency pairing. Figure 7 shows the order-parameter distribution for $p_{x}$-wave (a), $p_{y}$-wave (b), $d$-wave (c), and extended $s$-wave (d) pairings at $\Phi / \Phi_{0}=0.25$ in the $q=0$ sector. Compared with the zero flux case in Fig. 1, the $s$-wave component is highly suppressed and mainly located near the center of the loop edges. In contrast, the $d$-wave component is enhanced and may become the subdominant one of the order parameter. Particularly, the spin-singlet $s$ - and $d$-wave pairings both vanish in the diagonal directions, that is, spin-triplet even-parity pairing may be induced there by the spin rotational symmetry breaking. We checked the present system when $\Phi / \Phi_{0}=0.25$ and found that the spin-triplet $s$ and $d$-wave components both exist at the sample diagonals, 

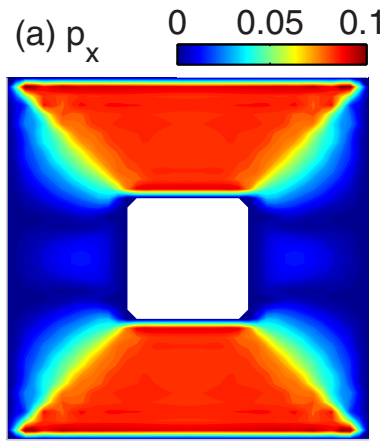

(c) $d$

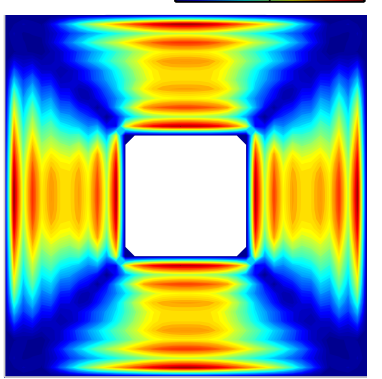

(b) $p$

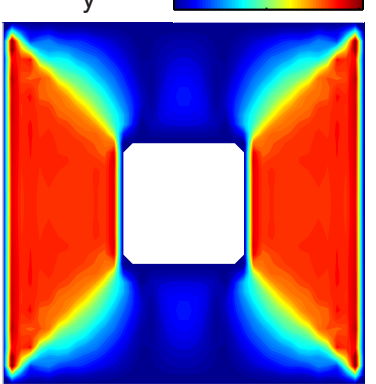

(d) $\mathrm{S}$

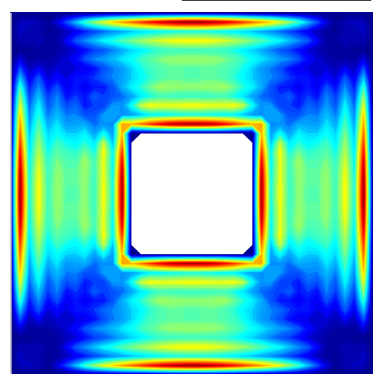

FIG. 7. (Color online) Contour plots of $p_{x}$-wave (a), $p_{y}$-wave (b), $d$-wave (c), and extended $s$-wave (d) pairings for a square $40 \times 40$ loop with an arm width $w=14$ at $\Phi / \Phi_{0}=0.25$ in the $q=0$ sector when $\mu=-1.5$.

especially at the corners. As a result, the LDOS feature at zero bias in Fig. 6 can be used to establish the odd-frequency pairing in the STM measurement.

In order to examine the spin current distribution related to the magnetization seen in Fig. 6(f), Fig. 8 gives the spatial profiles of $J_{\uparrow}$ (a) and $J_{\downarrow}$ (b) as well as $J_{c}$ (c) and $J_{s}$ (d) at $\Phi / \Phi_{0}=0.25$. Obviously, the spin-up and spin-down currents both show peaks at the outer corners, while their distributions near the inner corners behave differently. Notice that the persistent charge currents circulate with opposite directions in the current channels of the sample. In contrast to the currents flowing in one direction in Fig. 4(c), the charge currents with an opposite sign mainly appear near the inner edge of the sample, as clearly seen in Fig. 8(c). Moreover, in Fig. 8(d), we find that the oscillating feature on the diagonals in Fig. 4(d) is suppressed, especially near the outer corners. At the same time, the spin currents are enhanced inside the sample arms. This spatial variation of the spin current is identical to the evolution of the magnetization.

\section{MESOSCOPIC LOOP WITH DOMINANT $d$-WAVE PAIRING}

In this section, we would like to discuss the spontaneous magnetic moment and spin currents for a mesoscopic superconducting loop with dominant spin-singlet $d_{x^{2}-y^{2}}$-wave symmetry in the same spirit of the analysis performed in the previous section for the triplet case. In order to realize the mixed parity pairing in the system at zero temperature, the parameters are chosen to be $V=1.8, U=1.5$, and $\mu=-0.6$. The lower absolute values of the attractive interaction and chemical
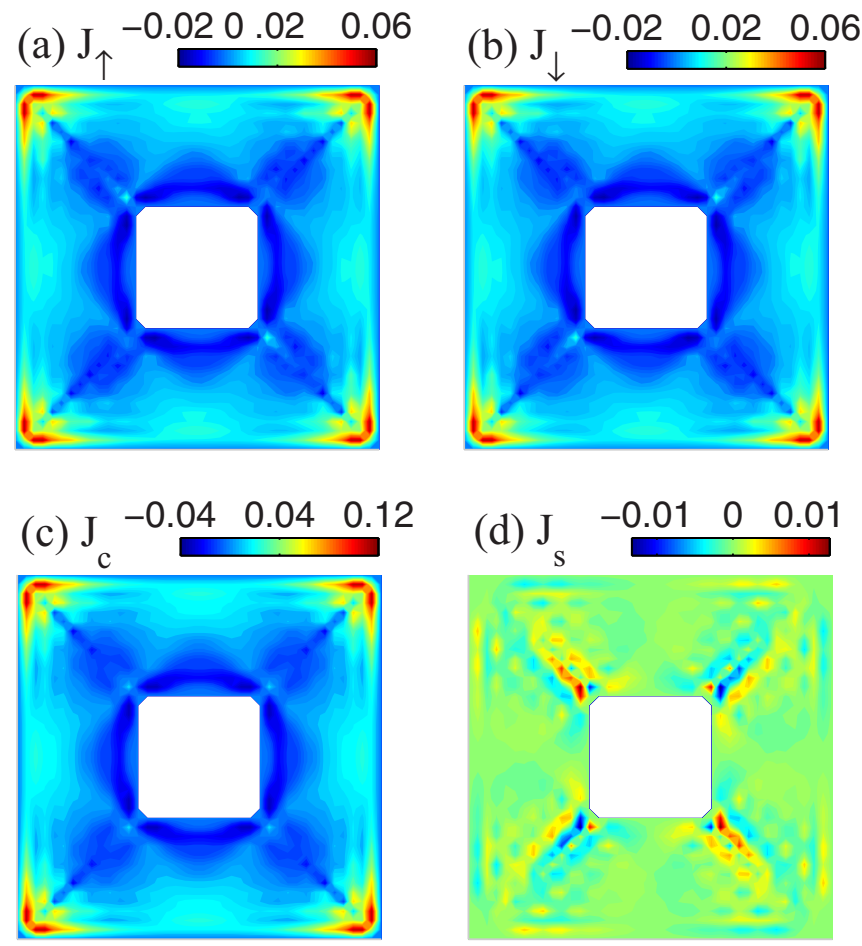

FIG. 8. (Color online) Contour plots of spin-polarized currents $J_{\uparrow}$ (a) and $J_{\downarrow}$ (b) as well as the charge current $J_{c}$ (c) and spin current $J_{s}(\mathrm{~d})$ for a square $40 \times 40$ loop with $w=14$ at $\Phi / \Phi_{0}=0.25$ in the $q=0$ sector when $\mu=-1.5$.

potential, compared with the previous triplet case, can lead to a higher total electron density. In this case the subdominant component of the order parameter develops in the spin-triplet $p$-wave channel, and the bulk $s$-wave component cannot emerge because of very low $|\mu|$.

In Fig. 9 we show the spatial profiles of order parameters for $d$-wave (a), extended $s$-wave (b), $p_{x}$-wave (c), and $p_{y}$-wave (d) pairings in the square loop at zero threaded magnetic flux. For the dominant $d$-wave superconducting order, we observe slight oscillations in the order-parameter profile. The $d$-wave order parameter is suppressed at the sample surface, thus inducing an extended $s$-wave component only near the inner and outer edges. For the chosen band structure, the subdominant $p$-wave component arises with a similar shape to the triplet case in Fig. 1. Thus, the coexistence of the singlet and triplet order parameters can be obtained again. It is noted that the $p$-wave order gradually disappears with decreasing $|\mu|$, and only $d$ wave pairing can be observed when $\mu \sim 0$.

The mixed pairing symmetries indicate the appearance of spontaneous magnetization due to the unequal densities of spin-up and spin-down electrons. Figure 10 displays the spatial variations of the zero-energy $\operatorname{LDOS} \rho_{\uparrow}(E=0)$ (a), $\rho_{\downarrow}(E=0)(\mathrm{b}), \rho_{\uparrow \downarrow}(E=0)(\mathrm{c})$, and the magnetization $m_{\mathbf{i}}$ (d) at zero flux. We can also observe the zero-energy peaks of LDOS on the sample's diagonal, resembling the triplet case in Fig. 2. That is, the OTE pairing may occur where the LDOS is peaked. However, in this case, the zero-bias conductance spectra for the spin-up and spin-down electrons are clearly distinct from one another, i.e., the zero-energy peaks in different diagonal directions are dominated by different 

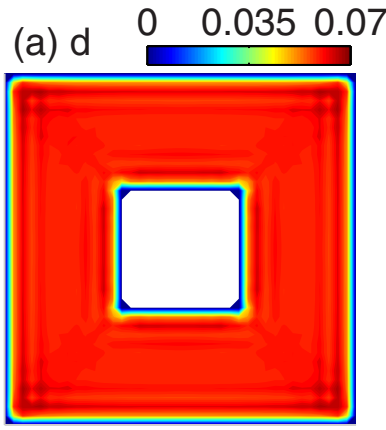

(c) $p_{x} \quad 0 \quad 0.02 \quad 0.05$

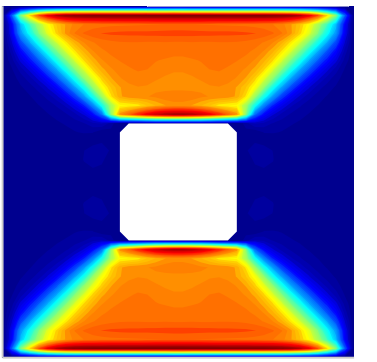

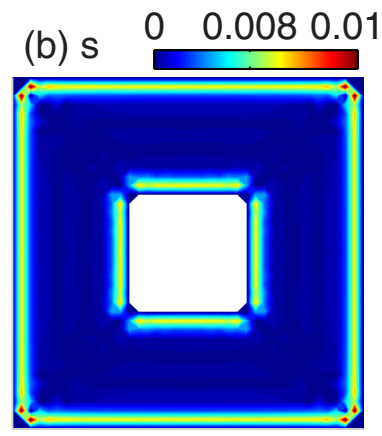
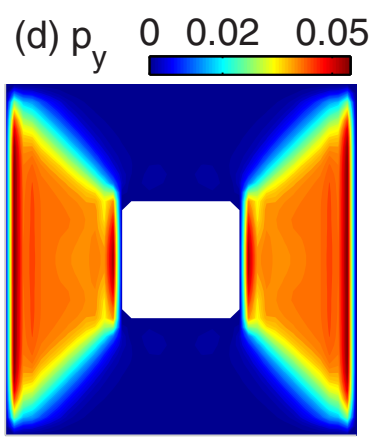

FIG. 9. (Color online) Contour plots of $d$-wave (a), extended $s$-wave (b), $p_{x}$-wave (c), and $p_{y}$-wave (d) pairings for a square $40 \times 40$ loop with an arm width $w=14$ at zero flux. The calculation is performed with $V=1.8, U=1.5, \mu=-0.6$, and $T=0$.

spin channels [see Figs. 10(a) and 10(b)]. As a consequence, the induced local magnetic moment, which is determined by the balance between spin-up and spin-down channels, shows a spin-dependent feature on the sample diagonals [see Fig. 10(d)].

(a)

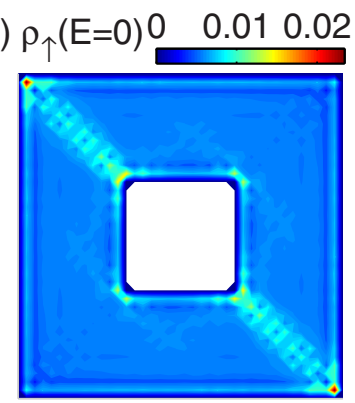

(c)

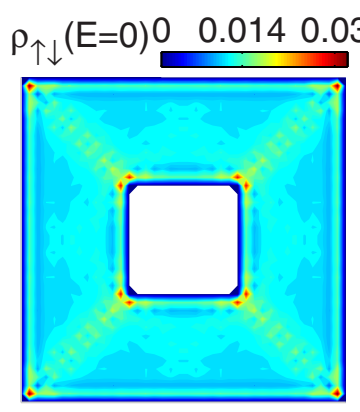

(b)
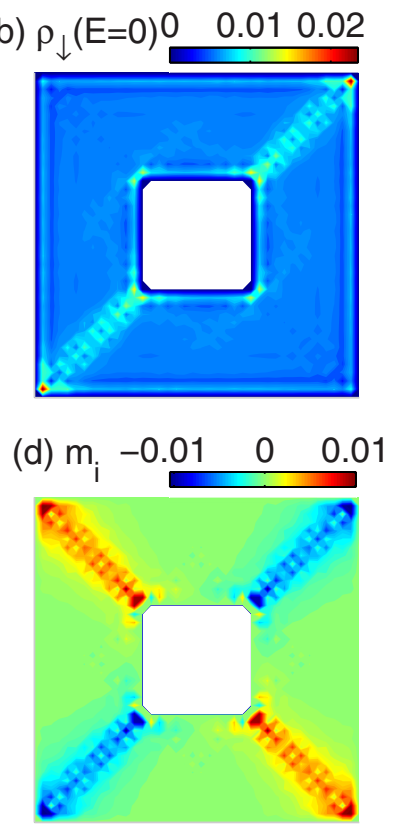

FIG. 10. (Color online) Contour plots of $\rho_{\uparrow}(E=0)(\mathrm{a}), \rho_{\downarrow}(E=$ $0)$ (b), $\rho_{\uparrow \downarrow}(E=0)(\mathrm{c})$, and $m_{\mathbf{i}}$ (d) for a square $40 \times 40$ loop with $w=14$ at $\Phi / \Phi_{0}=0$.

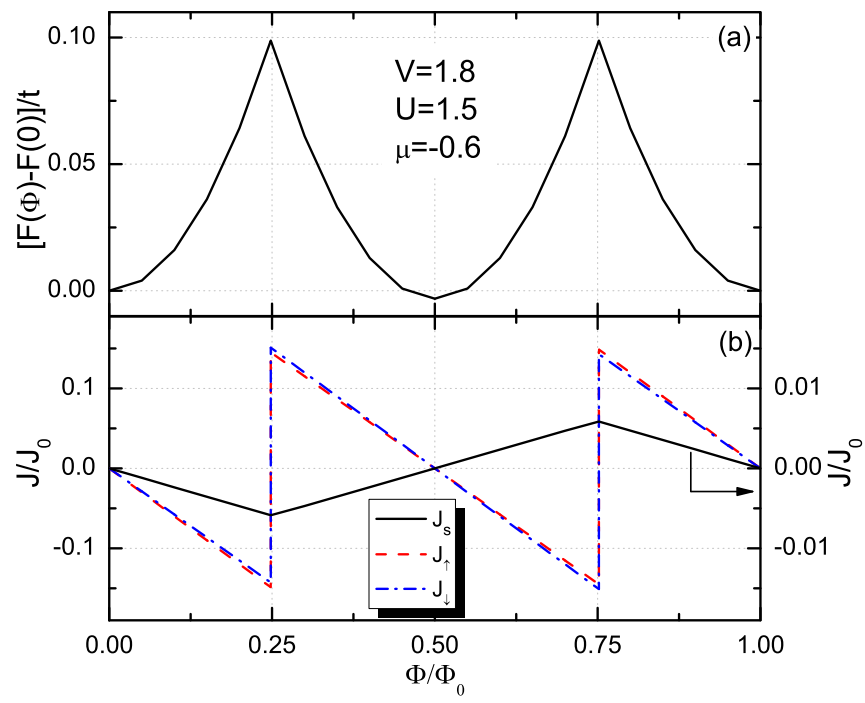

FIG. 11. (Color online) Total energy (a) and spin current $J_{s}$ (right-hand scale) and spin-polarized currents $J_{\uparrow}$ and $J_{\downarrow}$ (the left-hand scale) (b) for a square $40 \times 40$ loop with $w=14$ as a function of $\Phi$.

Next, we investigate the evolution of the total energy and the persistent charge and spin currents in the presence of a threaded magnetic flux. As discussed in Sec. III, besides the circulating charge current, the spin current can be induced by flux because of the broken equality of the spin-up and spin-down current components. For a mesoscopic loop with dominant $d$-wave pairing, the corresponding evolution of the total energy (a) and the charge and spin currents (b) with flux is plotted in Fig.11. Note that $J_{s}$ is right-hand scaled in Fig. 11(b). We find that the system exhibits a slight deviation from the $\Phi_{0} / 2$-periodic behavior, which is similar to previous studies in pure $d$-wave loops with weak pairing potential $[1,8]$. The energy parabolas in Fig. 11(a) reach a local minimum at $\Phi / \Phi_{0}=0$ and a global one at $\Phi=\Phi_{0} / 2$. Simultaneously, the flux value of the $q \rightarrow q+1$ jump tends to shift from $\Phi_{0} / 4$ toward a smaller one. Notice that this small deviation would disappear and the conventional $h c / 2 e$-periodic behavior of charge currents can be restored entirely for an enlarged attractive interaction $V$.

Interestingly, differently from the charge current, we always observe an $h c / e$-flux periodicity in the spin current even if the pairing strength is increased. As displayed by the solid curve in Fig. 11(b), the spin current $J_{s}$ is nearly continuous and does not show jumps with flux at the phase transition points between flux regimes belonging to different $q$ states. Notably, at the flux values with current jumps $J_{\uparrow(\downarrow)}$ flows oppositely, while $J_{s}$ does not change its sign due to the relative magnitude for $J_{\uparrow}$ and $J_{\downarrow}$ switching in different flux regimes. This phenomenon does not show up for the previous triplet case when the current jumps between the sectors with even and odd winding numbers [see Figs. 1(b) and 5(b)]. Moreover, Fig. 12 gives the spatial profiles of $J_{\uparrow}(\mathrm{a}), J_{\downarrow}(\mathrm{b}), J_{c}(\mathrm{c})$, and $J_{s}(\mathrm{~d})$ at $\Phi / \Phi_{0}=0.2$. We can find the deviation from the symmetric pattern of $J_{c}$ for the spin-polarized current $J_{\uparrow}$ and $J_{\downarrow}$. As a result, the spin current occurs. Corresponding to the magnetization profile in Fig. 10(d), the spin-up and spin-down electrons contribute respectively to the peaks of $J_{s}$ on different sample diagonals. 

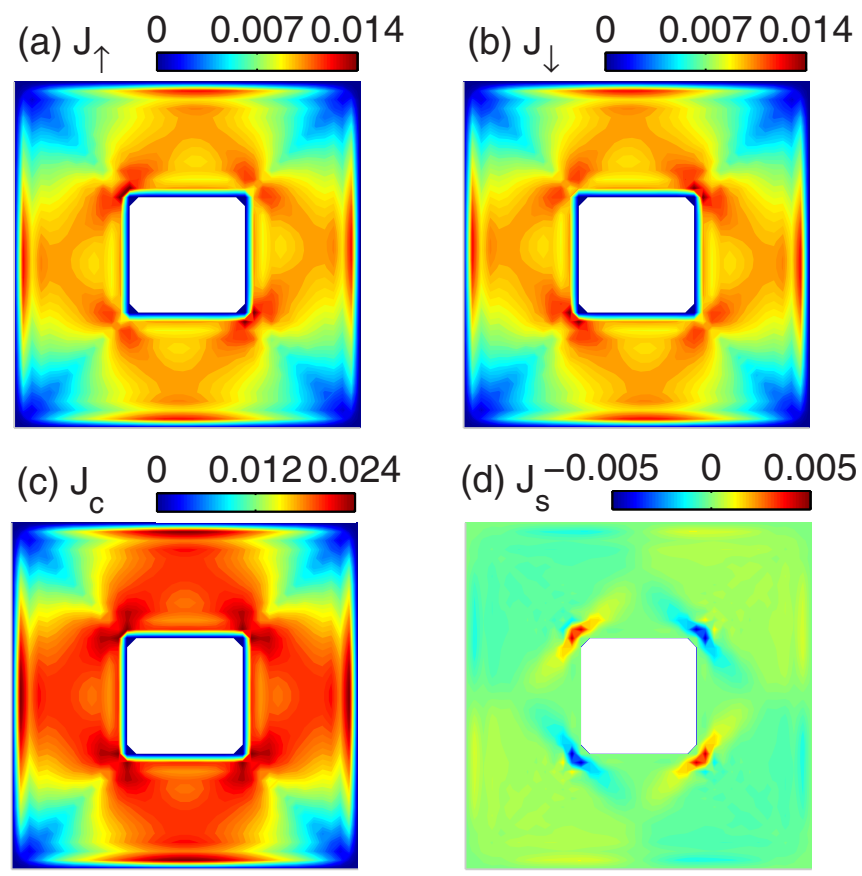

FIG. 12. (Color online) Contour plots of $J_{\uparrow}$ (a), $J_{\downarrow}$ (b), $J_{c}$ (c), and $J_{s}(\mathrm{~d})$ for a square $40 \times 40$ loop with $w=14$ at $\Phi / \Phi_{0}=0.2$ in the $q=0$ sector.

\section{CONCLUSIONS}

We have investigated the mixed pairing symmetry of order parameters and flux-induced spin current in mesoscopic superconducting loops by numerically solving the BdG equations self-consistently. It was found that the spin-triplet and spin-singlet superconducting states can coexist as the band structure is changed. Two types of samples with dominant $p_{x} \pm i p_{y}$-wave and $d_{x^{2}-y^{2}}$-wave symmetry were separately studied. A spontaneous magnetization can occur for both cases with mixed parity pairing due to the unequal densities of spin-up and spin-down electrons. The corresponding zerobias conductance spectra were given. When the threaded magnetic flux turns on, the flux-dependent charge current depicts periodic evolution. Interestingly, the spin current can also emerge because of the broken equality of the spin-up and spin-down current components. In the case of a mesoscopic loop with dominant triplet $p$-wave symmetry, we demonstrated that a slight change of the chemical potential may give rise to novel flux-dependent evolution of the ground-state energy and the magnetization. The pronounced saw-tooth-like patterns of the spin-polarized currents, corresponding to fractional flux periodicity, were induced due to the appearance of energy jumps in flux, accompanied with a steplike feature of the enhanced spin current. In particular, the odd-frequency pairing may appear on the sample diagonals at appropriate flux, which can be distinguished by the zero-energy LDOS in STM experiments. Simultaneously, the spin-polarized currents may flow with opposite directions near the inner and outer edges of the sample. Finally, in the case of a square loop with dominant singlet $d$-wave symmetry, we found that the spatial variations of the magnetization and the spin current display spin-dependent features on different sample diagonals. At the flux values where the current jumps between the sectors with even and odd winding numbers, we did not observe the sign changing of the spin current. Consequently, the flux-dependent evolution of the spin current always shows an $h c / e$ periodicity, differently from the triplet case. We expect that our predictions may provide useful guidance for futuristic applications in superconducting electronics and spintronics. Also, using the tight-binding model allows us to take certain aspects of the real band structure into account. Our theoretical calculations may be useful for some other systems, such as the noncentrosymmetric superconductors in which the singlet and the triplet components are intrinsically mixed [34]. For $\mathrm{Sr}_{2} \mathrm{RuO}_{4}$ with three electronic bands, one can restrict to one band only since one of the three bands, such as the two-dimensional $\gamma$ band, may dominate the superconducting properties [35-38]. For the superconductor-ferromagnet heterostructures, the groundstate phase diagram within the mean-field approximation was examined by Hirsch [39] in the uniform case with $U>0$ and $V=0$ (i.e., the repulsive Hubbard model). One can use this result to choose the parameters to realize the ferromagnetic state in the ferromagnet layer [23,24].

\section{ACKNOWLEDGMENTS}

This work was supported by the National Natural Science Foundation of China under Grants No. 61371020 and No. 61271163, by the Visiting Scholar Program of Shanghai Municipal Education Commission, and by the Flemish Science Foundation (FWO-Vl).
[1] F. Loder, A. P. Kampf, T. Kopp, J. Mannhart, C. W. Schneider, and Y. S. Barash, Nat. Phys. 4, 112 (2008).

[2] T.-C. Wei and P. M. Goldbart, Phys. Rev. B 77, 224512 (2008).

[3] V. Vakaryuk, Phys. Rev. Lett. 101, 167002 (2008).

[4] Y. S. Barash, Phys. Rev. Lett. 100, 177003 (2008).

[5] V. Juričić, I. F. Herbut, and Z. Tešanović, Phys. Rev. Lett. 100, 187006 (2008).

[6] F. Loder, A. P. Kampf, and T. Kopp, Phys. Rev. B 78, 174526 (2008).

[7] F. Loder, A. P. Kampf, T. Kopp, and J. Mannhart, New J. Phys. 11, 075005 (2009).
[8] G.-Q. Zha, M. V. Milosevic, S.-P. Zhou, and F. M. Peeters, Phys. Rev. B 80, 144501 (2009); 84, 132501 (2011).

[9] J.-X. Zhu and H. T. Quan, Phys. Rev. B 81, 054521 (2010).

[10] F. Loder, A. P. Kampf, and T. Kopp, Phys. Rev. Lett. 111, 047003 (2013).

[11] G.-Q. Zha, L. Covaci, F. M. Peeters, and S.-P. Zhou, Phys. Rev. B 90, 014522 (2014).

[12] V. Vakaryuk and V. Vinokur, Phys. Rev. Lett. 107, 037003 (2011).

[13] M. Sato and M. Kohmoto, J. Phys. Soc. Jpn. 69, 3505 (2000).

[14] T. Kuwabara and M. Ogata, Phys. Rev. Lett. 85, 4586 (2000). 
[15] J. Jang, D. G. Ferguson, V. Vakaryuk, R. Budakian, S. B. Chung, P. M. Goldbart, and Y. Maeno, Science 331, 186 (2011).

[16] X. Cai, Y. A. Ying, N. E. Staley, Y. Xin, D. Fobes, T. J. Liu, Z. Q. Mao, and Y. Liu, Phys. Rev. B 87, 081104(R) (2013).

[17] V. M. Edelstein, Phys. Rev. Lett. 75, 2004 (1995).

[18] L. P. Gorkov and E. I. Rashba, Phys. Rev. Lett. 87, 037004 (2001).

[19] V. V. Kabanov, Phys. Rev. B 69, 052503 (2004).

[20] B.-L. Gao and S.-J. Xiong, Phys. Rev. B 75, 104507 (2007).

[21] R. Micnas, J. Ranninger, and S. Robaszkiewicz, Rev. Mod. Phys. 62, 113 (1990).

[22] K. Kuboki, J. Phys. Soc. Jpn. 70, 2698 (2001).

[23] K. Kuboki and H. Takahashi, Phys. Rev. B 70, 214524 (2004).

[24] M. Cuoco, A. Romano, C. Noce, and P. Gentile, Phys. Rev. B 78, 054503 (2008).

[25] A. Romano, P. Gentile, C. Noce, I. Vekhter, and M. Cuoco, Phys. Rev. Lett. 110, 267002 (2013).

[26] P. G. de Gennes, Superconductivity of Metals and Alloys (Addison-Wesley, New York, 1994).

[27] S. L. Gnatchenko, A. M. Ratner, M. Baran, R. Szymczak, and H. Szymczak, Phys. Rev. B 55, 3876 (1997).
[28] J.-X. Zhu and C. S. Ting, Phys. Rev. Lett. 87, 147002 (2001).

[29] A. Ghosal, C. Kallin, and A. J. Berlinsky, Phys. Rev. B 66, 214502 (2002).

[30] G.-Q. Zha, H.-W. Zhao, and S.-P. Zhou, Phys. Rev. B 76, 132503 (2007).

[31] J. W. Harter, B. M. Andersen, J. Bobroff, M. Gabay, and P. J. Hirschfeld, Phys. Rev. B 75, 054520 (2007).

[32] Y. Tanaka, M. Sato, and N. Nagaosa, J. Phys. Soc. Jpn. 81, 011013 (2012).

[33] T. Yokoyama, Y. Tanaka, and A. A. Golubov, Phys. Rev. B 72, 052512 (2005); 75, 134510 (2007).

[34] A. B. Vorontsov, I. Vekhter, and M. Eschrig, Phys. Rev. Lett. 101, 127003 (2008).

[35] D. F. Agterberg, T. M. Rice, and M. Sigrist, Phys. Rev. Lett. 78, 3374 (1997).

[36] M. E. Zhitomirsky and T. M. Rice, Phys. Rev. Lett. 87, 057001 (2001).

[37] M. Takigawa, M. Ichioka, and K. Machida, Phys. Rev. B 65, 014508 (2001).

[38] J.-W. Huo and F.-C. Zhang, Phys. Rev. B 87, 134501 (2013).

[39] J. E. Hirsch, Phys. Rev. B 31, 4403 (1985). 